\title{
Management of cardiac tamponade during nivolumab of lung cancer with intrapericardial bleomycin: case report
}

\author{
Maiko Asai ${ }^{1}$, Yasuhiro Kato*,1,2, Shoko Kawai ${ }^{1}$, Kageaki Watanabe ${ }^{1}$, Makiko Yomota ${ }^{1}$, \\ Yusuke Okuma ${ }^{1}$, Yukio Hosomi ${ }^{1}$, Tsunekazu Hishima ${ }^{3}$ \& Tatsuru Okamura ${ }^{1}$ \\ ${ }^{1}$ Department of Thoracic Oncology \& Respiratory Medicine, Tokyo Metropolitan Cancer \& Infectious Diseases Center, Komagome \\ Hospital, Bunkyo, Tokyo, Japan \\ ${ }^{2}$ Department of Pulmonary Medicine \& Oncology, Graduate School of Medicine, Nippon Medical School, Tokyo, Japan \\ ${ }^{3}$ Department of Pathology, Tokyo Metropolitan Cancer \& Infectious Diseases Center, Komagome Hospital, Bunkyo, Tokyo, Japan \\ *Author for correspondence: Tel.: +81 033823 2101; y-kato@nms.ac.jp
}

Immuno-checkpoint inhibitor response and immune-related adverse events remain controversial issues. Managing pericardial effusion during programmed cell death 1 inhibitor treatment is challenging. Here, we report a case of successfully managed cardiac tamponade caused by nivolumab-induced pseudoprogression. A 62-year-old male diagnosed with advanced lung adenocarcinoma started on nivolumab. Seven days later, he experienced cardiac tamponade and required pericardiocentesis, and other lesions were larger on computed tomography. The patient's condition stabilized after pericardiocentesis. However, although the lesions other than pericardial effusion were reduced on chest $\mathrm{CT}$, cardiac tamponade recurred after 6 weeks. We considered that the case involved cardiac tamponade induced by pseudoprogression and administered intrapericardial bleomycin after pericardiocentesis. Thereafter, the patient was administered nivolumab for 7 months until disease progression.

First draft submitted: 2 January 2017; Accepted for publication: 21 January 2018; Published online:

7 February 2019

Keywords: cardiac tamponade $\bullet$ intrapericardial bleomycin $\bullet$ lung cancer $\bullet$ nivolumab $\bullet$ pericardial effusion $\bullet$ programmed cell death 1 inhibitor $\bullet$ pseudoprogression

Immune therapy is a new treatment option for cancer. Cytotoxic T-lymphocyte-associated protein 4 antibodies (ipilimumab and tremelimumab) programmed cell death 1 (PD-1) inhibitors (nivolumab and pembrolizumab), programmed-cell death ligand 1 inhibitors (atezolizumab, avelumab and durvalumab) are considered as new treatment options for certain types of cancer. Nivolumab developed as an immunoglobulin G4 antibody is a PD-1 inhibitor. Previous trials have revealed that nivolumab is superior to cytotoxic chemotherapy after platinumdoublet therapy in patients with recurrent non-small-cell lung cancer (NSCLC) [1]. Similarly, pembrolizumab and atezolizumab presented more efficacies of recurrent NSCLC patients $[2,3]$. The keynote 024 trial showed that pembrolizumab is superior to platinum-doublet chemotherapy in untreated NSCLC patients with higher tumor proportion score of PD-L1 [4]. Therefore, especially PD-1 inhibitors and PD-L1 inhibitors into immune therapy were considered as one of the treatment options for patients of NSCLC.

The evaluation of tumor responses after the initiation of PD- 1 inhibitor treatment is challenging because the response of tumors to nivolumab deviates from that of typical cytotoxic agents. Edema and inflammation resulting from immune cell infiltration caused by $\mathrm{PD}-1$ inhibitor treatment mimic tumor progression; this phenomenon is known as pseudoprogression [5]. A retrospective analysis reported that $2 \%$ of all patients with NSCLC experience pseudoprogression after the initiation of PD-1 inhibitor treatment [6].

Pericardial effusion causes cardiac tamponade and troublesome symptoms, such as dyspnea, loss of consciousness and shock, thereby worsening the performance status of such patients. Although the response evaluation criteria in solid tumors guideline (version 1.1) defines pericardial effusion as 'nonmeasurable', pericardial effusion with malignant tumors often involves clinical tumor progression [7]. However, pseudoprogression and immune-related adverse events (irAEs) should be considered as mechanisms of increasing pericardial effusion by PD-1 inhibitor 


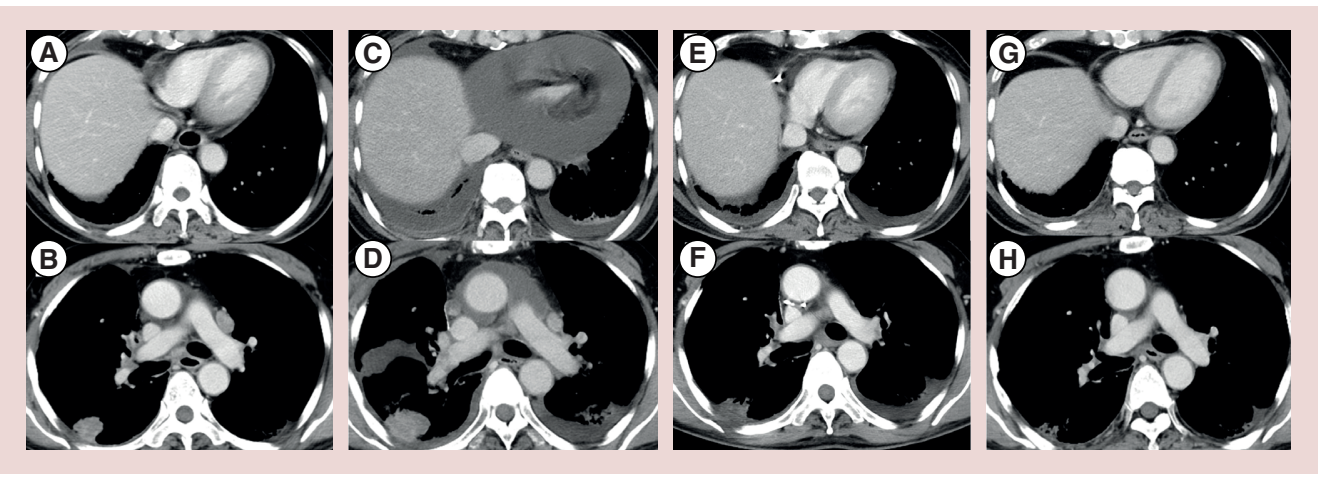

Figure 1. Imaging course of pericardial effusion and primary lesion. (A) Computed tomography (CT) performed before starting nivolumab showed mild pericardial effusion. (B) A $3.2 \times 2.5$-cm right inferior mass. (C) Chest CT at 7 days revealed increased pericardial effusion and (D) a $4.5 \times 3.1-\mathrm{cm}$ mass of the right inferior lobe growing larger than baseline. (E) Chest CT after the second pericardiocentesis revealed controlled pericardial effusion and (F) decreased size of the mass. (G) After 2 months, intracardiac bleomycin and nivolumab were restarted, and chest CT revealed that the mass was decreasing in size. (H) Pericardial effusion did not recur.

treatment without disease progression. Therefore, managing pericardial effusion after initiating nivolumab therapy is challenging but important for making clinical decisions. However, few literatures reported pericardial effusion after initiating nivolumab or other immune therapy.

Here, we report a case of successfully managed cardiac tamponade caused by nivolumab-induced pseudoprogression treated with intrapericardial bleomycin.

\section{Case report}

A 62-year-old male with a history of smoking presented with a tumor lesion in the right upper lobe of his lung. Transbronchial biopsy, computed tomography (CT) and positron emission CT for whole-body survey were performed for diagnostic purposes. The patient was diagnosed with stage IIB lung squamous cell carcinoma (cT3N0M0). Four cycles of S-1 plus cisplatin were initiated as neoadjuvant therapy. Right upper lobectomy was subsequently performed, and the patient was diagnosed with pT3N0M0 squamous cell carcinoma.

However, 8 months later, new lesions appeared in the right lower lobe with mediastinal lymphadenopathy of the right supraclavicular node and mild pericardial effusion. Biopsy of the right supraclavicular lymph node was performed. Tissue biopsy sample showed thyroid transcription factor-1 positive adenocarcinoma, and the patient was diagnosed with advanced lung adenocarcinoma as metachronous second primary lung cancer (Figure 1A \& B). Molecular analysis revealed negativity for EGF receptor mutation and anaplastic lymphoma kinase rearrangement as well as a PD-L1 tumor proportion score of $90 \%$. Because the patient refused to undergo cytotoxic chemotherapy and lung adenocarcinoma was developed immediately after the completion of platinum doublet therapy, nivolumab was initiated.

After 7 days, he presented to our hospital with dyspnea. Chest CT revealed that increased pericardial effusion, and his primary and lymph node lesions became larger (Figure 1C \& D). He went into shock, and his pulse pressure was $<20 \mathrm{mmHg}$. Emergency pericardiocentesis was performed to improve pericardial tamponade. Pericardial effusion cytology revealed malignant cells (Figure 2A). His general condition stabilized after pericardiocentesis, and he hoped to undergo observation without continuous nivolumab therapy. However, he experienced another cardiac tamponade with dyspnea 6 weeks after pericardiocentesis. After undergoing pericardiocentesis, cytology again revealed adenocarcinoma (Figure $2 \mathrm{~B}$ ). Because the lesions other than pericardial effusion were reduced on chest CT, pseudoprogression was considered as the pathogenesis of his pericardial effusion (Figure $1 \mathrm{E} \& \mathrm{~F}$ ). Although we proposed to surgically create a pericardial pleural window to control the pericardial effusion, the patient refused to undergo this procedure owing to its invasiveness. We administered intrapericardial bleomycin (bleomycin: $15 \mathrm{mg} /$ body) once and restarted nivolumab. Two months later, nivolumab was restarted, the tumor response was continuous without recurrence of pericardial effusion (Figure $1 \mathrm{G} \& \mathrm{H}$ ). After receiving nivolumab for 7 months without increasing pericardial effusion, nivolumab was replaced with pemetrexed owing to abdominal lymph node recurrence. He did not experience other irAE during nivolumab therapy. Figure 3 presents the patient's 


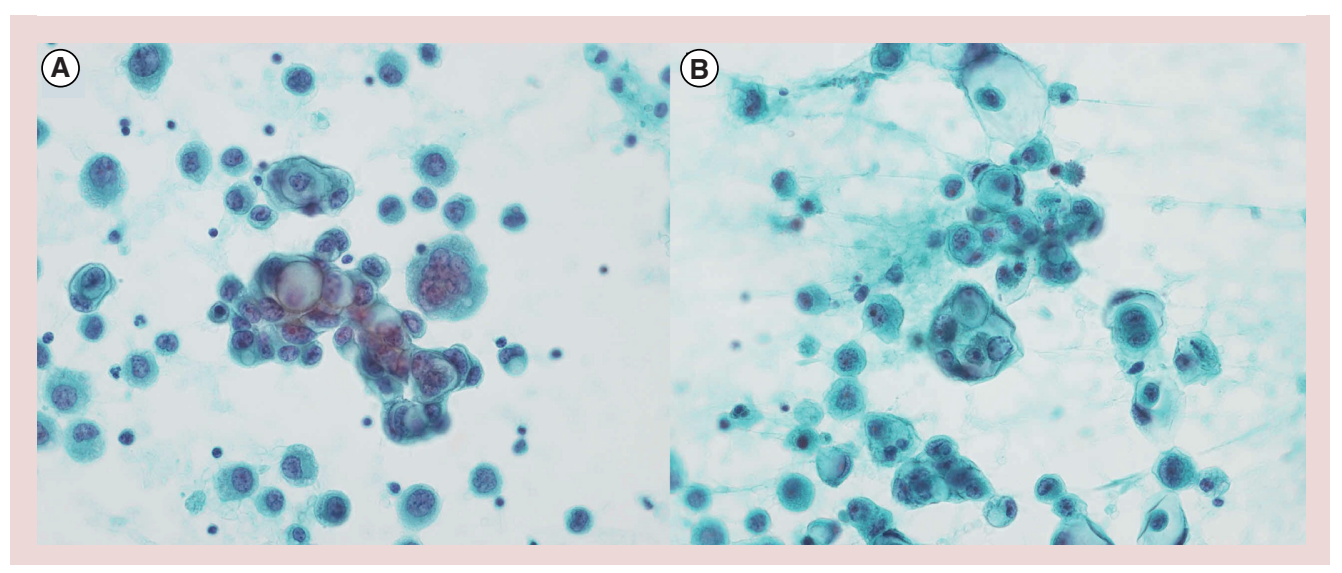

Figure 2. Cytolgies of pericardial effusion. (A) Cytology of pericardial effusion collected by first pericardiocentesis after nivolumab revealed adenocarcinoma (Papanicolaou stain). (B) Cytology of pericardial effusion collected by second pericardiocentesis after nivolumab revealed adenocarcinoma again (Papanicolaou stain).

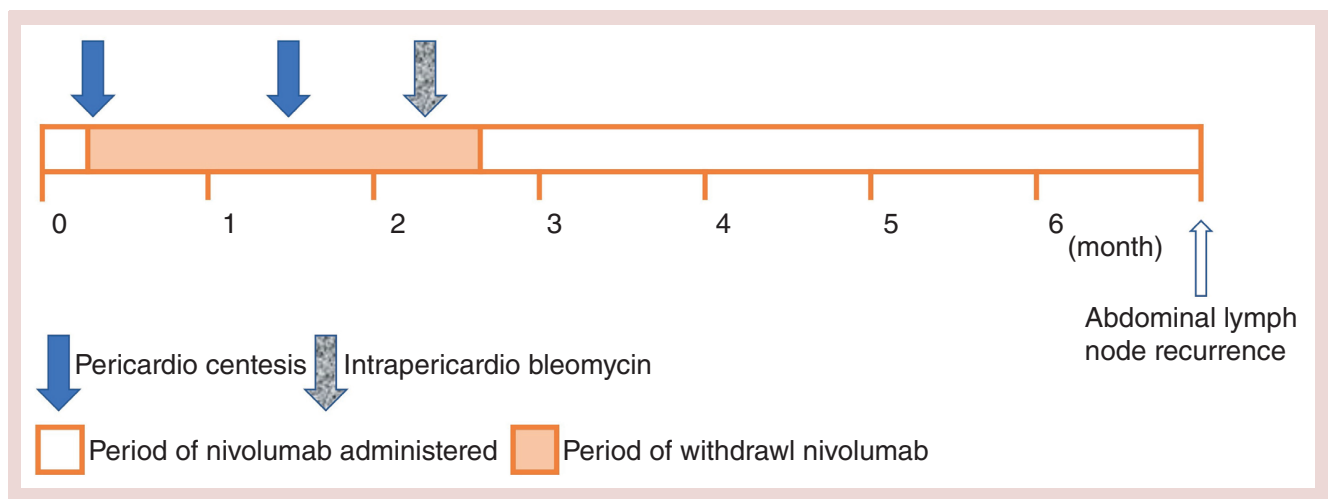

Figure 3. Clinical course of the patient after initiating nivolumab. After the withdrawal of nivolumab, 7 days after initiating nivolumab, he was diagnosed with cardiac tamponade. After he received pericardiocentesis the first time, his condition stabilized. However, cardiac tamponade recurred after 6 weeks. We administered intrapericardial bleomycin after pericardiocentesis. Thereafter, the patient was administered nivolumab for 7 months until recurrence of lesions in abdominal lymph node.

clinical course after nivolumab was restarted. Since the initiation of pemetrexed, no relapse of pericardial effusion has occurred.

\section{Discussion}

Some studies reported on pericardial effusion after the initiation of nivolumab therapy [8-11]. All cases in these previous literatures needed pericardiocentesis. In most cases, pericardial effusion presented as an irAE [8-10] and corticosteroids were administered in order to control cardiac for management of pericardial effusion [8-11]. Pericardial tamponade induced by pseudoprogression after immune therapy was rare. As far as we searched, only one study reported two patients in whom pericardial effusion presented pseudoprogression and required pericardiocentesis [11]. In this report, one patient required corticosteroid administration to control cardiac tamponade; in another patient, cardiac tamponade improved with the use of pericardiocentesis alone [11].

Because lymphocyte- and cytokine-induced inflammation reduced with corticosteroid treatment, the treatment was expected to improve the mechanisms of increasing pericardial effusion, pseudoprogression and irAEs. Some previous literatures reported effect of corticosteroid therapy during immune therapy. According to previous literature, corticosteroid therapy for irAE does not affect immunotherapy for cancer and that the discontinuation of immune therapy for irAE affects the outcome of immunotherapy $[12,13]$. On the other hand, one retrospective study suggested that corticosteroids decrease the efficacy of PD-1 inhibitors [14]. However, this research reported that 
only baseline corticosteroid therapy superior to $10 \mathrm{mg}$ per day prednisone is significantly associated with decreased progression-free survival of PD-1 inhibitors. Therefore, using corticosteroid for pericardial effusion induced by pseudoprogression should be considered as one of the useful treatment options.

On the other hand, in our case, we successfully controlled pericardial effusion with intrapericardial bleomycin without corticosteroid. Therefore, this case report shows the utility of intrapericardial bleomycin for managing pericardial effusion induced by pseudoprogression of immune therapy. As far as we searched, our manuscript was first case to report conducting intrapericardial bleomycin to manage pericardial effusion after immune therapy.

Intrapericardial bleomycin for malignant pericardial effusion has been hypothesized to reduce malignant pericardial cells with bleomycin. Prospective trials have been conducted on the utility of intrapericardial bleomycin. Kunitoh et al. reported effusion failure-free survival, referring to patient survival without the recurrence of malignant pleural or pericardial effusion in patients exhibiting primary control with intrapericardial bleomycin at a higher rate than with only pericardiocentesis [15].

In our patient, intrapericardial bleomycin was considered to interfere with increasing pericardial effusion for relapsed lymphocytic inflammation by reducing malignant cells in the pericardium; however, there remains some controversy regarding the underlying mechanism. For example, the mechanism of increasing pericardial effusion in our patient is debatable due to the lack of adequate pathological analyses (i.e., protein, glucose, cell count or cell block) and lymphocyte counts. In addition, if our patient presented with pseudoprogression, it would be possible to control cardiac tamponade with only pericardiocentesis without administering intrapericardial bleomycin. However, cardiac tamponade, which requires urgent treatment, causes shock and may lead to cardiac arrest. Our patient required two emergency pericardiocentesis. In the second pericardiocentesis, reduction of the other lesions was observed on CT and pericardial effusion increased causing cardiac tamponade, suggesting that intrapericardial bleomycin was required to control cardiac tamponade in our patient. In our patient, intrapericardial bleomycin produced long-term efficacy of nivolumab and effusion failure-free survival after nivolumab replaced pemetrexed as anticancer drug.

Other medical procedures to resolve these clinical problems include surgical creation of a pericardiopleural window [16]. However, such surgeries require general anesthesia and are invasive; thus, our patient refused to undergo the procedure. Intrapericardial bleomycin presented safety in previous literature [15]. It should be considered that intrapericardial bleomycin is one of the treatment options for the management of immune therapy-related pericardial effusion.

\section{Conclusion}

This study shows that pseudoprogression should be considered a mechanism for increasing pericardial effusion after the initiation of PD-1 inhibitor treatment. Intrapericardial bleomycin was a useful treatment option for controlling pericardial effusion-induced pseudoprogression. This study should help in making future clinical decisions.

Financial \& competing interests disclosure

Y Okuma and Y Hosomi received honoraria from Bristol-Myers Squibb and Ono Pharmaceutical CO. The authors have no other relevant affiliations or financial involvement with any organization or entity with a financial interest in or financial conflict with the subject matter or materials discussed in the manuscript apart from those disclosed.

Medical writing support was provided by Enago and was self-funded by the authors.

Informed consent disclosure

The authors state that they have obtained verbal and written informed consent from the patient/patients for the inclusion of their medical and treatment history within this case report.

Ethical conduct of research

The authors state that they have obtained appropriate institutional review board approval or have followed the principles outlined in the Declaration of Helsinki for all human or animal experimental investigations. In addition, for investigations involving human subjects, informed consent has been obtained from the participants involved. 
Summary points

- Programmed cell death 1 (PD-1) inhibitor response and immune-related adverse events remain controversial issues. Previous studies reported the following three mechanisms of pericardial effusion after the initiation of PD-1 inhibitor treatment: tumor progression, pseudoprogression and immune-related adverse events.

- Therefore, managing pericardial effusion during PD-1 inhibitor treatment is challenging. Here, we report a case of successfully managed cardiac tamponade caused by nivolumab-induced pseudoprogression.

- A 62-year-old male diagnosed with advanced lung adenocarcinoma was started on nivolumab. After 7 days, he experienced cardiac tamponade and required pericardiocentesis.

- Cytology of the pericardial effusion revealed that the adenocarcinoma and other lesions were larger on computed tomography. However, the patient's condition stabilized after pericardiocentesis, and he hoped to discontinue therapy with any anticancer agents.

- However, cardiac tamponade recurred after 6 weeks. Chest computed tomography revealed responses of other lesions without pericardial effusion. Pericardiocentesis was again performed and cytology revealed adenocarcinoma.

- We considered that this case involved cardiac tamponade induced by pseudoprogression and administered intrapericardial bleomycin.

- Thereafter, the patient was administered nivolumab for 7 months without increasing pericardial effusion until recurrence of lesion in abdominal lymph node.

- To the best of our knowledge, this is the first reported case demonstrating the utility of intrapericardial bleomycin for cardiac tamponade induced by pseudoprogression after the initiation of nivolumab.

\section{References}

Papers of special note have been highlighted as: $\bullet$ of interest

1. Horn L, Spigel DR, Vokes EE et al. Nivolumab versus docetaxel in previously treated patients with advanced non-small-cell lung cancer: two-year outcomes from two randomized, open-label, Phase III trials (CheckMate 017 and CheckMate 057). J. Clin. Oncol. 35(35), 3924-3933 (2017).

2. Rittmeyer A, Barlesi F, Waterkamp D et al. Atezolizumab versus docetaxel in patients with previously treated non-small-cell lung cancer (OAK): a Phase III, open-label, multicenter randomized controlled trial. Lancet 389(10066), 255-265 (2017).

3. Herbst RS, Baas P, Kim DW et al. Pembrolizumab versus docetaxel for previously treated, PD-L1-positive, advanced non-small-cell lung cancer (KEYNOTE-010): a randomized controlled trial. Lancet 387(10027), 1540-1550

4. Reck M, Rodríguez-Abreu D, Robinson AG et al. Pembrolizumab versus chemotherapy for PD-L1-positive non-small-cell lung cancer. N. Eng. J. Med. 375(19), 1823-1833

5. Wolchok J D, Hoos A, O'Day $S$ et al. Guidelines for the evaluation of immune therapy activity in solid tumors: immune-related response criteria. Clin. Cancer Res. 15(23), 1078-0432 (2009).

- Presents observation of immune therapy and tumor response. Interestingly, infiltration of T-lymphocyte to melanoma nodules after immune therapy is shown in this article.

6. Katz S I, Hammer M, Bagley S J et al. Radiologic pseudoprogression during Anti-PD-1 therapy for advanced non-small cell lung cancer. J. Thorac. Oncol. 13(7), 978-986 (2018).

7. Eisenhauer E A, Therasse P, Bogaerts J et al. New response evaluation criteria in solid tumors: revised RECIST guideline (version 1.1). Eur. J. Cancer 45(2), 228-247 (2009).

8. Kushnir I, Wolf I. Nivolumab-induced pericardial tamponade: a case report and discussion. Cardiology 136(1), 49-51 (2017).

- Reports a case of advanced squamous cell carcinoma presented cardiac tamponade after nivolumab. In this case, cytology of pericardial effusion did not show malignant cells and the author considered the mechanism of pericardial effusion as immune-related adverse events. Prednisone was initiated for the management of pericardial effusion in this case.

9. Nesfeder J, Elsensohn A N, Thind M, Lennon J, Domsky S. Pericardial effusion with tamponade physiology induced by nivolumab. Int. J. Cardiol. 222, 613-614. (2016).

- Reports a case of a patient with non-small-cell lung cancer (NSCLC) who presented with cardiac tamponade after nivolumab initiation. In this case, placement of pericardial window was conducted for management of pericardial effusion.

10. Vittorio A, Sharma R, Siejka D, Bhattarai K, Hardikar A Recurrent pericardial effusion while receiving nivolumab for metastatic lung adenocarcinoma: case report and review of the literature. Clin. Lung Cancer 19(5), e717-e720. (2018).

- Reports a case of adenocarcinoma presented with malignant pericardial effusion. In this case, although pericardiocentesis and creation of a pericardial window were performed several times, each time the patient experienced pericardial tamponade after nivolumab initiation. Interestingly, after withdrawal of immune therapy, pericardial effusion did not increase. 
11. Kolla B C, Patel M R. Recurrent pleural effusions and cardiac tamponade as possible manifestations of pseudoprogression associated with nivolumab therapy-a report of two cases. J. Immunother. Cancer 4(1), 80. (2016).

- This article first reports pseudoprogression of pericardial effusion in two cases. Interestingly, one case needed only pericardiocentesis, and in the other case corticosteroid for management of pericardial effusion was initiated.

12. Horvat TZ, Adel NG, Dang T-O et al. Immune-related adverse events, need for systemic immunosuppression, and effects on survival and time to treatment of failure in patients with melanoma treated with ipilimumab at Memorial Sloan Kettering Cancer Center. J. Clin. Oncol. 33(28), 3193-3198, (2015)

13. Schadendorf D, Wolchok JD, Hodi FS et al. Efficacy and safety outcomes in patients with advanced melanoma who discontinued treatment with nivolumab and ipilimumab because of adverse events: a pooled analysis of randomized Phase II and III trials. J. Clin. Oncol. 35, 3807-3814, (2017)

14. Arbour K C, Mezquita L, Long N et al. Impact of baseline steroids on efficacy of programmed cell death-1 and programmed death-ligand 1 blockade in patients with non-small-cell lung cancer. J. Clin. Oncol. 1; 36(28), 2872-2878 (2018).

15. Kunitoh $\mathrm{H}$, Tamura $\mathrm{T}$, Shibata $\mathrm{T}$ et al. randomized trial of intrapericardial bleomycin for malignant pericardial effusion with lung cancer (JCOG9811). Br. J. Cancer 100(3), 464-469. (2009).

16. Vairkus PT, Hermann HC, Lewinter MM. Treatment of malignant pericardial effusion. JAMA 272, 59-64 (1994). 